\title{
Tissue Processing, Our Experience in the Lab
}

\author{
Sangeeta $\mathrm{M}^{* 1}$, Raja Parthiban ${ }^{2}$,Shilpa Naik ${ }^{3}$,Sahana B N ${ }^{4}$ \\ 1,3,4 (Dept of Anatomy, MVJ Medical College and Research Hospital) \\ ${ }^{2}$ (Dept of Pathology,, MVJ Medical College and Research Hospital)
}

\begin{abstract}
All the techniques employed for the study of histology bring an insight into the details inside the cell with the use of microscope. The simplest and most common method in the study of histology is prepa ration of sections. Tissues are exposed to the series of reagents that fix, dehydrate, clear, infiltrate with final embedding in a medium which provides support for the tissue. The quality of structural preservation is determined by the choice of reagents and exposure times to the reagents during processing. Each step in the tissue processing is important from the procurement of the specimen, selection of sample, determining the appropriate protocols and reagents to be used. This paper compares certain methods of tissue processing and the results obtained through them.
\end{abstract}

Key words: Tissue,processing,staining,fixing,dehydration,clearing.

\section{Introduction}

Aim of tissue processing is to remove water from the tissue and replace it with the medium that solidifies to allow thin sections to be cut. Biological tissues must be supported in a hard matrix to allow sufficiently thin sections to be cut, typically $5 \mu \mathrm{m}$ thick for light microscopy. For light microscopy paraffin wax is most frequently used. Since it is insoluble in water, the main constituent of biological tissue, water must first be removed in the process of dehydration. Samples are transferred through baths of progressively more concentrated ethanol to remove the water. This is followed by hydrophobic clearing agent such as xylene to remove the alcohol and finally molten paraffin wax the infiltration agent, which removes the xylene. After the block is obtained, sectioning can be done using a steel knife mounted in a microtome to cut $4 \mu \mathrm{m}$ thick tissue sections which are finally mounted on a glass slide. Biological tissue has little inherent contrast in either light or electron microscope. Staining is employed to give both contrast to the tissues as well as high lighting particular features of interest. Haematoxylin and eosin are the most commonly used light microscopical stain in histology and histopathology. Haematoxylin ,a basic dye stains nuclei blue due to affinity to nucleic acid. Eosine,an acidic dye stains cytoplasm pink ${ }^{1}$.

\section{Aims and objectives}

To compare three different methods of tissue processing and to decipher which method yields a good slide.

Liver and lung tissue from white mice

\section{Materials}

$10 \%$ formalin

Propanol of different grades $(30 \%, 50 \%, 70 \%, 90 \%$,absolute)

Xylene

Wax bath

Paraffin wax with ceresin

Rotary Microtome

Floatation bath

Glass slides

Egg albumin

Harris haematoxylin and eosine stain

Cover slips

\section{Methods}

Three different methods of tissue processing were used to compare the outcome of $\mathrm{H}$ and $\mathrm{E}$ staining on the lung and liver tissue.

After obtaining lung and liver tissues from the white mice, they were thoroughly washed in running tap water and were cut into small pieces of 3 to $4 \mathrm{~mm}$ thickness and immersed in 10\%formaline for 2 days. 


\section{Method 1}

Fixation: $10 \%$ formalin for two days

Dehydration : $30 \%$ alcohol-30min

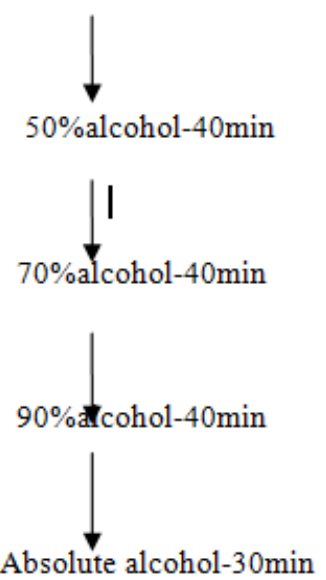

Clearing:

Mixture1-(25ml xylene:75ml absolute alcohol)-30min

Mixture 2-(50ml xylene:50ml absolute alcohol)-30min

Mixture3-(75ml xylene:25ml absolute alcohol)-30min

Pure clearing agent-till tissue becomes transparent ${ }^{(2)}$

\section{Method 2}

Fixation: $10 \%$ formaline for two days Dehydration and clearing

$70 \%$ alcohol- $12 \mathrm{hrs}$
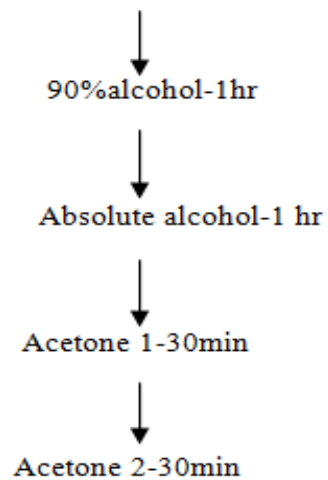

Acetone $2-30 \mathrm{~min}$

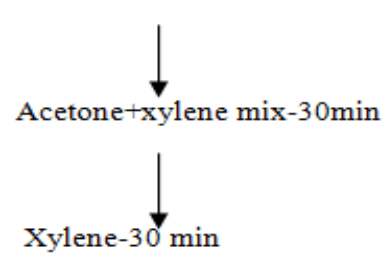

\section{Method 3}

Fixation: $10 \%$ formaline for two days Dehydration: 


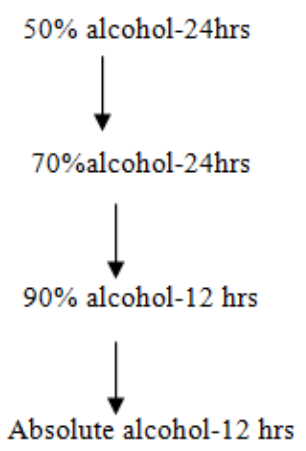

Clearing

Xylene-till the tissue becomes transparent

\section{Embedding}

Once the tissues become transparent ,they are transferred to the paraffin wax contained in the wax bath for embedding(temperature $60 \circledast \mathrm{C}$ ). There are four vessels containing the paraffin wax in which tissues are transferred successively.

$$
\begin{aligned}
& \text { Paraffin I-1hr } \\
& \text { Paraffin II-30min } \\
& \text { Paraffin III-30min } \\
& \text { Paraffin IV-30min }
\end{aligned}
$$

\section{Block preparation and tissue cutting}

Once the tissues are embedded with the paraffin wax ,tissue blocks are prepared with the help of brass moulds and section cutting is done with the help of rotary microtome. Thin ribbons are transferred to floatation bath. Glass slides coated with egg albumin are used to take out the thin sections from the floatation bath. These sections are stained using following staining method.

\section{Staining procedure $(\mathrm{H} \text { and } \mathrm{E} \text { staining })^{3}$}

For deparaffinising the tissue -Xylol-5 to 10min

For hydration-

\section{Absolute alcohol-2min}

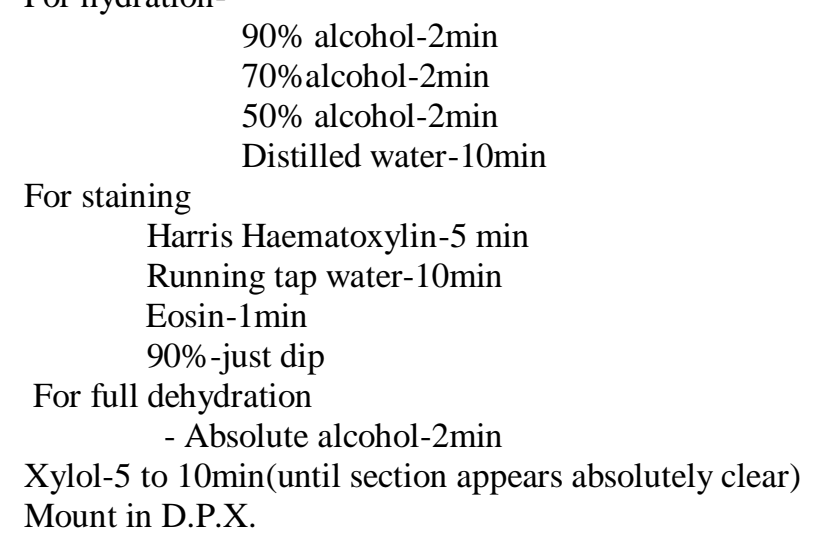




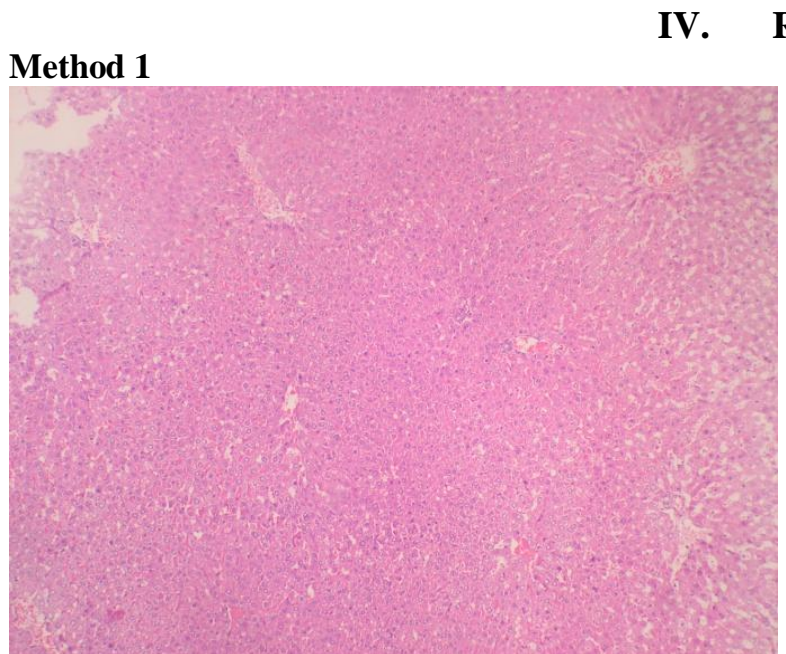

Liver

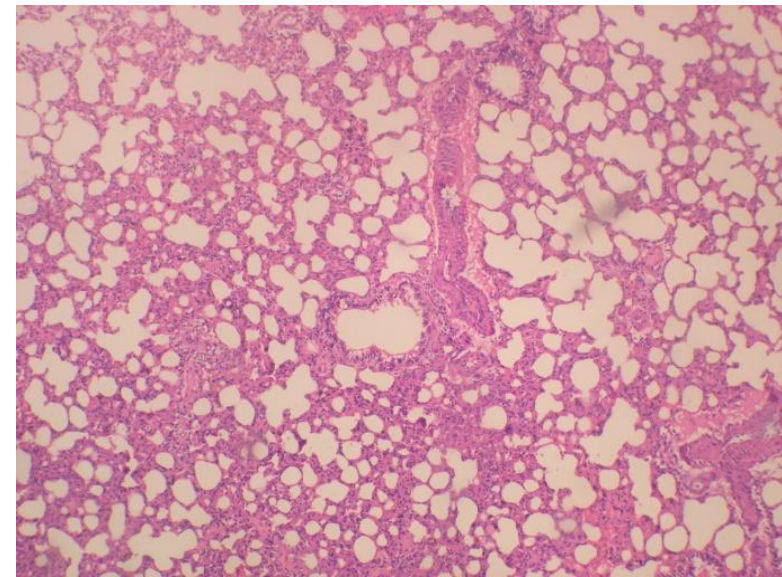

Lung

Method 2

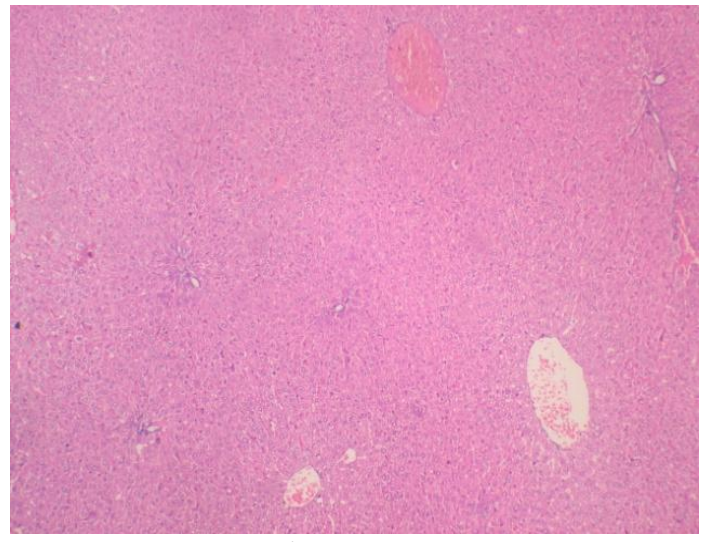

Liver

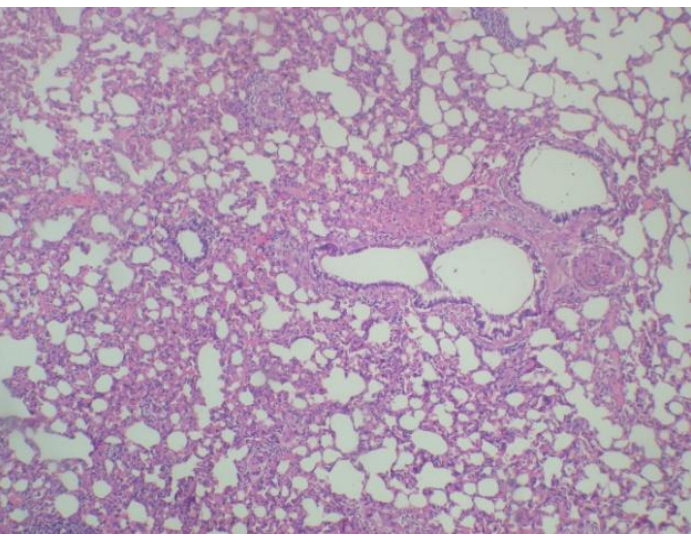

Lung

Method 3

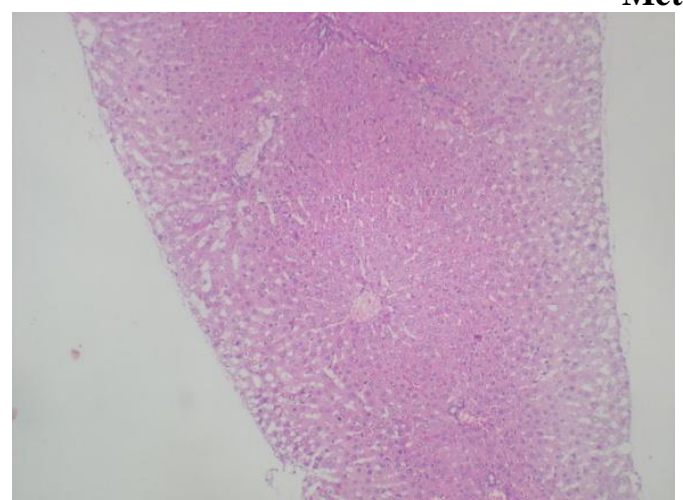

Liver

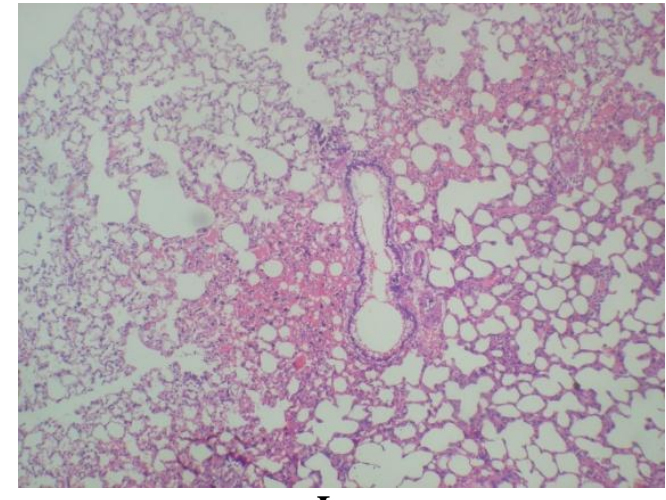

Lung

The best result was obtained in method $\mathbf{1}$ in terms of cellular architecture and staining by H\&E.

\section{Discussion}

Fixation in all the three methods (method 1,2 and 3) was obtained by immersing the tissue in $10 \%$ formalin for two days. The difference was apparent in the procedure of dehydration, where in, in method 1, slow and gradual dehydration was done by transferring the tissue through graded concentrations of alcohol( $30 \%$ alcohol for 30min,50\% alcohol for 40min,70\%alcohol for $40 \mathrm{~min}, 90 \%$ alcohol for $40 \mathrm{~min}$,absolute alcohol for 30min).Gradual dehydration has the advantage of preventing shrinkage of tissues ${ }^{4}$. In method three although gradual dehydration was attempted, we omitted keeping the tissue in 30\% alcohol and proceeded directly, to $50 \%$ alcohol. $50 \%$ alcohol for $24 \mathrm{hrs}, 70 \%$ alcohol for $24 \mathrm{hrs}, 90 \%$ alcohol for $24 \mathrm{hrs}$, absolute alcohol for 
12hrs).Keeping the tissues too long in higher dilutions of alcohol makes the tissues brittle and difficult to cut subsequently, also keeping the tissue in lower dilutions of alcohol should be avoided as it will macerate the tissue. ${ }^{5}$ This could be the reason why method 3 did not give good result. The common practice of taking out the tissue out of alcohol and then placing the tissue in the clearing agent is faulty. The reason being that alcohol escapes from the surface of tissue into the air quicker than the clearing agent can get into it. This causes tissue shrinkage, which could have happened in method 2 and $3{ }^{6}$

\section{Conclusion}

To conclude .although the method of fixation was common in all the three methods, dehydration, clearing and embedding differed in the three methods. All the three methods of tissue processing ended with the common staining of haematoxylin and eosin. The best result was obtained through method 1 probably because of slow dehydration and appropriate method of clearing.

\section{References}

[1]. Culling C.F Handbook of histopathological and histochemical techniques. $3{ }^{\text {rd }}$ Ed; . (1974) Butterworth and Co Ltd.

[2]. D.R.Singh Principles \& Techniques in Histology Microscopy and Photomicrography. $1^{\text {st }}$ Ed ; (2003) CBS Publiations.

[3]. Lena T. Spencer and John D Bancroft. Tissue Processing Theory and Practice of Histological Techniques.6 th Ed. Elsevier Publications

[4]. M.I.Cox. Expeimental Mol Pthology.2006.Vol 80;183.

[5]. Carlson F.L..Histotechnology A self instrumental text. $3^{\text {rd }}$ Ed 2007.Chicago: ASCP Press

[6]. Stehen Gallik. Tissue Processing\& Histological stains 2009 (Internet) Available from histologyyolm.stevegalik.org 